\title{
ROLE OF NONSTATIONARY COLLISIONAL DYNAMICS IN DETERMINING NITRIC OXIDE LIF SPECTRA
}

\author{
John W. Daily \\ Department of Mechanical Engineering, University of Colorado, Boulder \\ Wolfgang G. Bessler, Christof Schulz \\ Physikalisch-Chemisches Institut, Universität Heidelberg \\ Volker Sick \\ Department of Mechanical Engineering, University of Michigan \\ Thomas B. Settersten \\ Combustion Research Facility, Sandia National Laboratories
}

\begin{abstract}
Quantitative analysis of laser-induced fluorescence signals of nitric oxide requires properly accounting for the effects of temperature, collisions, and laser and detection properties on the measured signals. Over the past years, LIF properties of nitric oxide were studied for a range of conditions, from well-controlled burners to internal combustion engines. The knowledge gained from these studies has been compiled into a model and software tool that now allows the computation of LIF spectra/signals for given experimental conditions. However, the excitation dynamics are treated in a simplified fashion. Here we discuss the role of nonstationary excitation dynamics on the LIF signal utilizing a comprehensive, time dependent, multi-level rate equation model.
\end{abstract}

\section{Introduction}

Since the development of lasers in the 1960s, laser spectroscopy has become an important technique in fundamental and applied research in chemistry and physics. Laser techniques have found broad application in combustion research [1]. They allow the remote measurement of physical (pressure, temperature), chemical (species concentrations) and gas dynamical (flow velocities) parameters [2,3]. Laser-induced fluorescence (LIF) is an important technique to help understand nitric oxide (NO) formation in both practical combustion systems and laboratory flames. Investigations aim at developing engineering solutions to minimize NO effluent, as well as developing and validating chemical kinetic models of NO formation and destruction. fluorescence signals requires a detailed understanding of the spectroscopy of the probed species and of the collisional dynamics that take place during the laser excitation process. This involves not only a quantummechanical description of the electronic structure and its interaction with electromagnetic radiation (term energies, transition strengths), but also a description of the laser-induced fluorescence process itself (population dynamics, intra- and intermolecular energy transfer). Furthermore, the influence of experimental boundary conditions (pressure, temperature, chemical composition of the bath gas, laser energy, pulse length and shape, detector characteristics) must be understood. Models of different complexity have been used to help understand the influence of and interactions between this variety of parameters.

For practical application, the spectroscopic background knowledge must be made accessible in a simple form to the experimentalist, who needs to convert measured LIF intensities to concentrations, temperatures, or pressures. Elsewhere [4] we have described a flexible model and software tool for the computation of LIF intensities, LIF excitation spectra, LIF emission spectra, and tables of temperature and pressure dependence of LIF signals. It also allows fitting simulated LIF excitation spectra to experimental data in order to evaluate temperatures and laser line widths from the measured spectra. However, the accuracy of the model is limited to some degree by oversimplification of the excitation dynamics module, which at present is based on a three-level, steady-state analysis. Here we explore the importance of non-steady behavior on the LIF signal characteristics.

The quantitative interpretation of laser-induced 


\section{NO Excitation Dynamics}

The energy level diagram for NO is shown in Figure 1. LIF diagnostics have been constructed by pumping from the X-state to both the A and B-states, although pumping to the A-state is preferable due to the longer excitation wavelengths required. This reduces problems with attenuation of laser light and LIF signals and complex electronic energy transfer.

In modeling the excitation process one solves a set of time dependent rate equations,

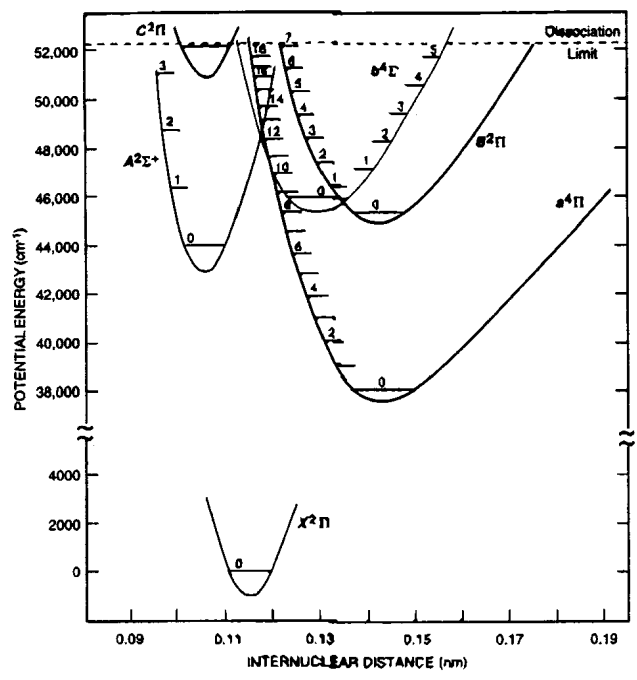

Figure 1 - NO Energy Level Diagram

one for the population $N_{i}$ of each quantum state $i$ considered. The equations take on the generalized form

$$
\frac{d N_{i}}{d t}=\sum_{j \neq i}\left(Q_{j i}+W_{j i}\right) N_{j}-\sum_{j \neq i}\left(Q_{i j}+W_{i j}\right) N_{i}-Q_{l o s t} N_{i}
$$

Here, the first term represents the rate at which collisions $\left(Q_{j i}\right)$ and radiative transitions $\left(W_{j i}\right)$ populate state $i$, the second term the rate at which state $i$ is depopulated by collisions and radiative transitions, and the final term $\left(Q_{\text {lost }}\right)$ the sum of all process that depopulate state $i$ to states not considered in the model. The collisional terms include rotational energy transfer (RET), vibrational energy transfer (VET) and electronic energy transfer (EET) to and from states considered in the model. The radiative terms are typically dominated by laser induced stimulated transitions, as spontaneous emission is overwhelmed by the collisional rates. The loss term can result from collisional or radiative transitions, including pre-dissociation or ionization.
Of critical concern in modeling the excitation dynamics are the collisional rates that dominate the response to excitation. At the present time, while collisional phenomena have been extensively studied, there are still no complete sets of collisional rates available for the practical situations faced in interpreting LIF data. One must review the literature for each target molecule, and, using considerable judgment regarding the quality of published data and theories, assemble a rate model that hopefully proves adequate to the challenge of data interpretation. In spite of the uncertainty in rates, some general conclusions are warranted for diatomic molecules like NO. First, it is generally the case that rotational energy transfer (RET) is the fastest of the possible energy transfer processes. Quenching, or electronic energy transfer (EET) rates vary considerably, depending on the target molecule and its collision partners. It is most likely that EET does not take place directly to the ground vibrational state, although there is so far no experimental evidence available. Finally, vibrational energy transfer (VET) rates are relatively slow compared to RET and EET, especially in the ground electronic state.

The picture that emerges is as follows. When laser excitation takes place, molecules from the lower laser coupled level are excited to the upper laser coupled level. Once there, they can radiate directly, or change rotational or vibrational level as a result of RET or VET and then radiate. They can also undergo quenching, either directly or following RET or VET. Finally, the molecule can dissociate or be ionized by the absorption of a second photon. Those molecules that collisionally change electronic state are generally lost for the duration of the laser pulse and sample time, as ground electronic state VET is such a slow process. (Dissociated and ionized molecules are definitely lost.) Meanwhile, as the lower laser coupled state population is depleted, RET will cascade population into that state as a result of the detailed balance requirement. (VET is too slow to contribute.) This scenario is clearly transient in nature, and to fully model the fluorescence signal one needs a transient model and accurate RET rates in the ground electronic state, and RET, VET, EET and pre-dissociation rates for the excited electronic state.

Because of the complexity of modeling the full molecule and a lack of rate data, the rate equations have been solved by numerous workers for a variety of simplified models. Many of these are discussed in the review by Daily [3] including steady state and transient two, three and four level models. For example, LIFSim [4] uses a three-level model in which the ground vibrational level is divided into a laser coupled level and a bath level. The third level is the laser-coupled excited state. An assumption, commonly made, is that the 
population in the lower laser-coupled state remains in its equilibrium ratio to the vibrational bath population. This reduces the problem to a two-level system that is easily solved. In this case, the excited state population is given by

$$
N_{A-\text { state }}=\frac{\frac{W f_{B}(T)}{(W+Q+A)}}{1+\frac{W f_{B}(T)}{(W+Q+A)}} N_{N O}
$$

where $W$ is the laser excitation rate, $f_{B}(T)$ the Boltzmann ratio for the lower laser coupled state, $Q$ the collision de-excitation or quenching rate for the excited state, and $A$ the spontaneous emission rate for the excited state. This solution displays saturation behavior, but does not allow for ground state hole burning which can be important in some cases. In addition to missing physics, one must often use average or effective rates. For example, at higher pressures it is almost impossible to excite a single transition and several excited state rotational levels will be populated. An important question is how well such simple solutions predict actual behavior.

\section{The NO Model}

To explore the implications of the various simplifying assumptions made in the implementation of the steady-state three-level and other simple LIF models, we have developed a comprehensive, detailed, transient model of NO excitation dynamics. The model solves the individual rate equations for all quantum states of the $\mathrm{X}$-state $\mathrm{v}=0-2$ and $\mathrm{A}$-state $\mathrm{v}=0$ and 1 vibrational manifolds including fully resolved spinorbit and parity states; over 950 energy levels are included. The spectral model includes all twelve major A$\mathrm{X}$ absorption and emission branches and takes into account laser and line broadening when calculating the excitation rate and line broadening when calculating the LIF emission spectra. The model can be used to calculate the time-dependent excitation dynamics and absorption spectra, excitation spectra and LIF emission spectra. Any of the spectra can be integrated over the pulse to obtain pulse averaged results and the peak hight of the transient signal can be identified and recorded. The LIF emission spectra can be convoluted with the appropriate filter or spectrometer transfer function to predict laboratory observed spectra or wavelength integrated signals.

To calculate the excitation dynamics properly the model requires term energies, transition wavelengths and probabilities, absorption line-broadening parameters, and all appropriate collisional rate constants.

\section{A. Term energies}

Term energies are calculated from theoretical expressions that are fit to experimental data. The ground, or X electronic state of $\mathrm{NO}$ has quantum numbers of spin orbital momentum $S=1 / 2$ and orbital angular momentum $\Lambda=1$, and its term symbol is thus

$$
{ }^{2} \Pi_{1 / 2,3 / 2}
$$

where the subscripts take into account the fact that the total electronic angular momentum $\Omega=\Lambda+S$ can take on the two values $1 / 2$ and $3 / 2$ based on the orientation of $\Lambda$. Thus the degeneracy of this state is four, and both spin-orbit and Lambda doubling occurs. For the ground vibrational level of the $\mathrm{X}$-state the spin-orbit constant $A \cong 119.82 \mathrm{~cm}^{-1}$ and the Lambda doubling constant $q \cong 0.0117 \mathrm{~cm}^{-1}$. Therefore, Lambda doubling is quite small compared to spin-orbit splitting. The X-state behaves more like Hund's case (a) for small $J$ and Hund's case (b) at larger $J$. As a result, most descriptions use the case (b) language to describe the rotational structure. The two spin-orbit split states are labeled

$$
\begin{aligned}
& F_{1}(N): J=N+1 / 2 \\
& F_{2}(N): J=N-1 / 2
\end{aligned}
$$

Here $F_{1}$ refers to the $\Omega=3 / 2$ state and $F_{2}$ to the $\Omega=1 / 2$ state. Each of the two spin-orbit states is further split because of Lambda doubling. To describe the term values for the X-state we use expressions from Pine et al. [5] with data from Amiot et al. [6]. Both are based on IR studies, meaning that only one electronic state is involved. Furthermore, Pine combines his data with Amiot's. The results for $\mathrm{v}=1$ and 2 states seem to be the best available. Pine and Amiot's fits agree with each other to within a wavenumber for the $\mathrm{v}=0,1$ and 2 states up to $\mathrm{J}$ about 60.5 .

The A-state of NO has $S= \pm 1 / 2$ and $\Lambda=0$. The term symbol for this state is

$$
{ }^{2} \Sigma^{+}
$$

The plus indicates the fact that the eigenfunction of this state is symmetric with respect to reflection about any plane that contains the inter-nuclear axis. The degeneracy is two, and spin-rotation splitting occurs. The ${ }^{2} \Sigma^{+}$ state behaves like Hund's case (b). The resulting two spin-rotation states are thus also labeled 


$$
\begin{aligned}
& F_{1}(N): J=N+1 / 2 \\
& F_{2}(N): J=N-1 / 2
\end{aligned}
$$

although for $\boldsymbol{N}=0$ there is no splitting of the levels. Parity is also important for the ${ }^{2} \Sigma^{+}$state. The elf notation of Brown et al. [7] is followed. Most workers have used the term expressions based on the developments of Hund [8], Van Vleck [9] and Mulliken [10] with a second order correction for centrifugal distortion. We use the Freedman and Nicholls' [11] fit as they made systematic use of the Engleman et al. [12] absorption data for the $(0,0)$ and $(1,0) \gamma$ bands.

Because of spin-orbit splitting and Lambda doubling, there is no single index labeling of the energy level of NO. Therefore, in our computer code, we assign each level an index based on sorting the levels by increasing energy. The energy and degeneracy of each level is stored in a matrix which also contains $n$ (the electronic energy level index), $v, J, \Omega$ and a parity index. In that way, each level can be uniquely identified.

\section{B. Transition Wavelengths and Probabilities}

From the term energies the transition energies of the NO A-X system (the so-called $\gamma$ bands) can be calculated using the selection rules of ${ }^{2} \Sigma-{ }^{2} \Pi$ dipole transitions. The selection rules lead to 12 branches for each $J$ ' value. The laser line/absorption line convolution integrals are calculated for the excitation rate matrix for all twelve branches.

For the calculation of transition strengths, vibrational oscillator strengths are taken from Laux and Kruger $[13,2]$; these values are based on ab initio calculations and potential energy curves obtained with recent spectroscopic constants. Ground-state energies, transition energies, and Einstein A and B coefficients were calculated and stored for each branch for ground-state rotational quantum numbers of $J$ " $\leq 50.5$, ground-state vibrational levels of $v^{\prime \prime}=0-11$, and excited-state vibrational levels of $v^{\prime}=0$ and 1 .

\section{Collisional line broadening and shifting}

Data for collisional line broadening and shifting for NO A-X transitions at elevated temperatures is limited. Chang, DiRosa and Hanson measured coefficients for $\mathrm{H}_{2} \mathrm{O}, \mathrm{O}_{2}, \mathrm{Ar}, \mathrm{N}_{2}$ and $\mathrm{NO}$ at room temperature in a static cell $[14,15]$ and for $\mathrm{H}_{2} \mathrm{O}, \mathrm{O}_{2}, \mathrm{Ar}$ and $\mathrm{N}_{2}$ at temperatures up to $2800 \mathrm{~K}$ in shock tubes [14,16]. They used cw dye laser absorption spectroscopy. Vyrodov et al. [17] performed room temperature measurements for
$\mathrm{N}_{2}$, Ar, and $\mathrm{He}$ at pressures up to 5 bar using LIF.

For calculation of the line shape, the broadening $(2 \gamma)$ and shifting $(\delta)$ coefficients and the temperature exponents are taken directly from literature $[14,16]$. Since data for all species other than $\mathrm{O}_{2}, \mathrm{H}_{2} \mathrm{O}$ and $\mathrm{N}_{2}$ are not available, the $\mathrm{N}_{2}$ coefficients are used for other colliders (especially the "missing" majority species $\mathrm{CO}_{2}$ ).

\section{Collisional Rates}

For the work reported here, the RET rates for the ground state were taken from Islam, Smith and Alexander [18]. Their data were then fit to a simple energy gap model that satisfies detailed balance. No parity changing or spin-orbit state changes are allowed in this preliminary case. The RET rate model is the same for all three vibrational levels treated, consistent with literature findings that RET rates are unaffected by vibrational state in the $\mathrm{X}$ electronic state. RET rates for the A-state are taken from Ebata et al. [19] using their exponential gap fit and insuring that detailed balance is satisfied. In both the $\mathrm{X}$ and A states, estimates of the total RET cross sections were made for species lacking direct data. VET rates for the X-state are taken from Wysong [20]. It is assumed for these calculations that angular momentum is preserved, that is there is no change in J when VET takes place. This is not exactly correct, but VET is so slow in the X-state, that during the $10 \mathrm{nsec}$ laser pulse essentially no VET takes place. VET rates for the A-state are taken from the summary in Wysong [20] or estimated by analogy. A-state quenching rates are calculated using the "harpoon" quenching model from Paul et al. [21], and future work will incorporate the recent measurements of the temperature-dependent quenching cross sections for major flame species [22].

\section{E. Photoionization}

At high laser irradiances photoionization [2325] from the A-state can be a significant population sink. The ionization rate can be expressed as

$$
W_{\text {ion }}=\frac{\sigma_{I} E}{h v}
$$

where $\sigma_{I}$ is the photoionization cross section, $E$ the laser irradiance, and $h v$ the photon energy. Zacharias and co-workers determined that the cross section is approximately $7 \times 10^{-19} \mathrm{~cm}^{2}$ for excitation via the $(0-0)$ band. We use their value in the code. 


\section{Numerical Calculations}

To study the effect of transient collisional processes on the excitation dynamics of $\mathrm{NO}$, calculations were carried out corresponding to a case of practical interest, typical high pressure combustion burned gas conditions. We ran a set of simulations for a mixture of $\mathrm{CO}_{2}, \mathrm{H}_{2} \mathrm{O}$ and $\mathrm{N}_{2}$ corresponding to stoichiometric combustion of methane. The mixture temperature was held at $1500 \mathrm{~K}$ and the NO concentration was 10 ppm. In all the calculations the irradiance was treated as spatially uniform, that is we did not include beam profile effects in this study, nor did we include laser or LIF absorption. (This was purposefully done to focus on the excitation dynamics. The model can handle both effects.) The laser was set at $226.034 \mathrm{~nm}$, a wavelength commonly used to avoid $\mathrm{O}_{2}$ interference [26].

\section{A. Excitation Rate}

The issue of excitation rate is important because of the balance between the desire to obtain a large LIF signal and the increased complexity of data reduction as saturation is approached. The excitation rate per molecule for a given radiative transition is given by the expression

$$
W_{12}(t)=\int_{0}^{\infty} B_{12} E_{v}(v, t) \phi(v) d v
$$

where $E_{\imath}(v, \tau)$ is the laser spectral irradiance. For the calculations presented here, we use a simple linear/exponential function for the laser temporal shape. $E_{\nu}(v, \tau)$ is written as

$$
E_{v}(v, t)=E_{0} \phi_{L}(v) f(t)
$$

where $E_{0}$ is the laser fluence $(\mathrm{J} / \mathrm{m} 2), \phi_{L}(v)$ is normalized laser line shape, and

$$
f(t)=\frac{t}{t_{p}^{2}} \exp \left(-\frac{t}{t_{p}}\right)
$$

is the normalized laser temporal pulse shape. We use a Gaussian function for the laser line shape with a linewidth of $0.3 \mathrm{~cm}^{-1} . t_{p}$ is set to one nanosecond.

In practice, because of line overlap and pressure broadening it is almost impossible to pump a single absorption transition and thus a single ro-vibrational state. As mentioned above, the calculations were carried out using a laser line center position of 226.034 $\mathrm{nm}$. This overlaps several lines including the $\mathrm{P}_{1}(23.5)$, $\mathrm{Q}_{1}(14.5), \mathrm{P}_{21}(14.5), \mathrm{Q}_{2}(20.5)$ and $\mathrm{R}_{12}(20.5)$. The $\mathrm{ab}-$ sorption spectrum in the range of the laser frequency is shown in Figure 2. As can be seen, the spectral features broaden and shift with pressure, and multiple lines are excited. The increase of excitation rate with pressure is due to an increased NO number density (the excitation rate per molecule actually decreases with increasing pressure because of line broadening.)

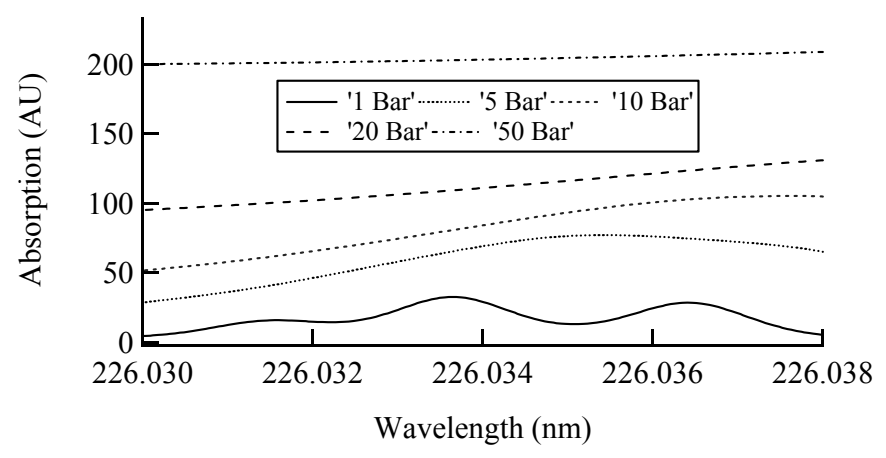

Figure 2 - Absorption spectrum in region close to laser wavelength $(226.034 \mathrm{~nm})$.

It is typical to compare the excitation rate, $W$, with the saturation rate $W^{S}$. This is because as the rate increases and saturation effects begin to appear, the dynamics change considerably and data reduction increases in complexity. However, for a molecular system like NO, it is quite difficult to define a saturation excitation rate. Therefore, most workers use the two level saturation rate, which is [3]

$$
W_{12}^{S}=\frac{Q_{21}+A_{21}}{1+g_{1} / g_{2}}
$$

where $Q_{21}$ is the quenching rate, $A_{21}$ is the spontaneous emission rate, and $g_{1}$ and $g_{2}$ are the degeneracies of state 1 and 2 respectively. For our base case at 1 Bar, this rate is about $4.7 \times 10^{8}$ per second. A typical $\mathrm{ND}: Y A G$ pumped dye laser system is capable of delivering pulse energies of about $500 \mu \mathrm{J}$ over a pulse of several nanoseconds with linewidth of about $0.3 \mathrm{~cm}^{-}$ ${ }^{1}$. The excitation rate is approximately

$$
W_{12} \sim B_{12} \frac{E_{p}}{A \Delta t} \varphi_{v}
$$

where $E_{p}$ is the pulse energy, $A$ the beam cross sectional area, $\Delta t$ the pulse width and $\phi_{v}$, the convolution integral. For a 3 nanosecond pulse with $0.3 \mathrm{~cm}^{-1}$ linewidth, the saturation rate corresponds to a beam fluence $\left(E_{p} / A\right)$ of about $10 \mathrm{~J} / \mathrm{m}^{2} .500 \mu \mathrm{J}$ focused to a $25 \mu \mathrm{m}$ spot size is a fluence of about $10^{6} \mathrm{~J} / \mathrm{m}^{2}$. Therefore, it is possible to 
achieve a high degree of saturation. However, at high levels of irradiance other processes such as photoionization and breakdown become important. In general, for point measurements the beam is focused to a diameter of about $150 \mathrm{~mm}$ and for PLIF to a sheet about $250 \mathrm{~mm}$ by $2 \mathrm{~cm}$. At $500 \mathrm{~mJ}$, these cases correspond to maximum beam fluences of about $30,000 \mathrm{~J} / \mathrm{m}^{2}$ and $100 \mathrm{~J} / \mathrm{m}^{2}$ respectively. As we explore the excitation dynamics keep these numbers in mind.

\section{B. Base Case Calculations}

As a base case we used the rates as described above, a laser fluence of $1000 \mathrm{~J} / \mathrm{m}^{2}$, and assumed that EET from the A-state was lost entirely during the laser pulse. As the laser intensity grows during the pulse, the populations of the ro-vibrational states in the Astates that are directly excited increase. In the absence of RET, these would be the only states populated and they would subsequently decay by radiation or collisional de-excitation. However, RET rates are fast enough that a significant amount of rotational redistribution takes place. This is illustrated in Figure 3, which shows the normalized rotational state population distribution $4 \mathrm{nsec}$ into the laser pulse for two pressures; 1 and 20 atmospheres.

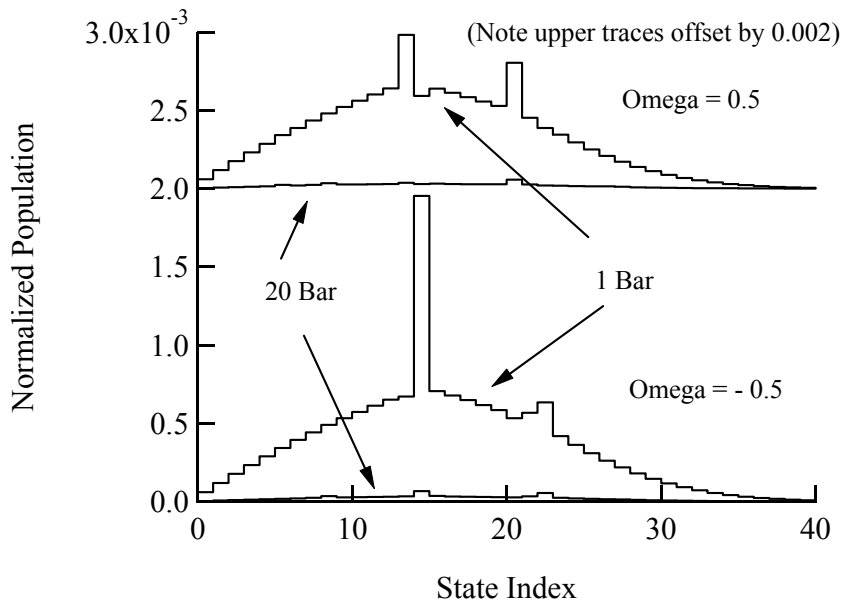

Figure 3 - A-state Rotational Population Distribution (4nsec); EET all Lost

(In all the Figures labeled "Normalized Population", the populations are normalized on the total initial NO number density. The "State Index" is the unique identifier for each energy level.) As can be seen, the population of the laser coupled states are elevated above those of the other states. Note that since RET rates scale with pressure, this redistribution occurs at the higher pressure as well. Corresponding to the laser excited states are the transition coupled ground ro-vibrational states. When the excitation rate is sufficiently high, one would expect hole burning in the ground state. For the excitation rate used in this calculation, there is significant hole burning at 1 atmosphere, but far less at 20 atmospheres as the RET rate has increased with respect to the excitation rate. This is illustrated in Figure 4. The total population in the $\mathrm{v}^{\prime}=0$ state is plotted as a function of time in Figure 5 with pressure as the parameter. As expected the normalized population decreases with pressure due to the increasing competition from quenching. (The absolute population scales with the pressure.)

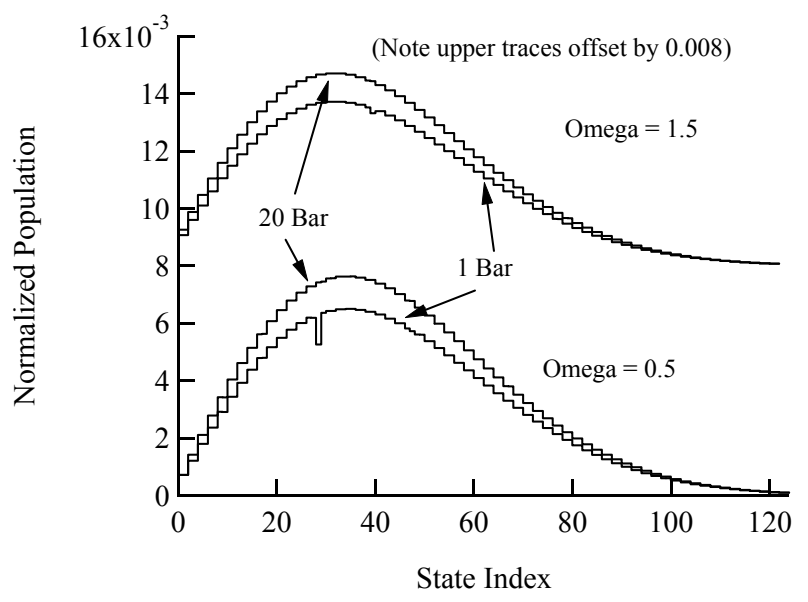

Figure 4 - X-state Rotational Population Distribution (4nsec); EET all Lost

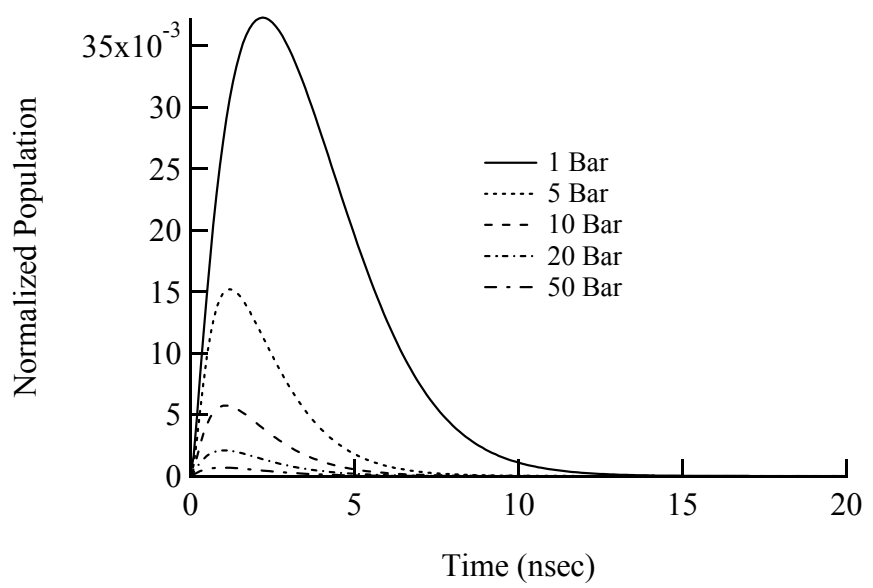

Figure 5 - A-state v' $=0$ Normalized Population-Time History; EET all Lost

Finally, we calculated (Figures 6 and 7) the saturation behavior as the laser irradiance was varied over five orders of magnitude. The "integrated signal" shown in Figure 6 is the spectrally integrated LIF signal averaged over the entire $20 \mathrm{nsec}$ experiment. The "peak" signal shown in Figure 7 is at the spectrally integrated LIF signal evaluated at the peak of the output signal pulse. These are signals commonly used in field 
experiments. As can be seen, at the higher irradiances the signals do appear to be approaching saturation. Note that as the pressure increases, saturation is delayed due to the faster collision rates. This causes the lower pressure curves to eventually cross over the higher pressure curves.

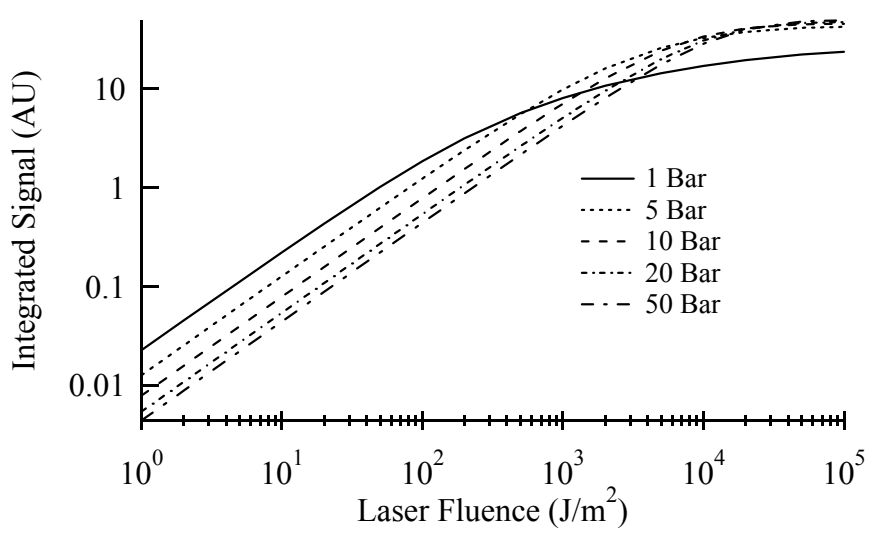

Figure 6 - Integrated LIF Signal; EET all Lost

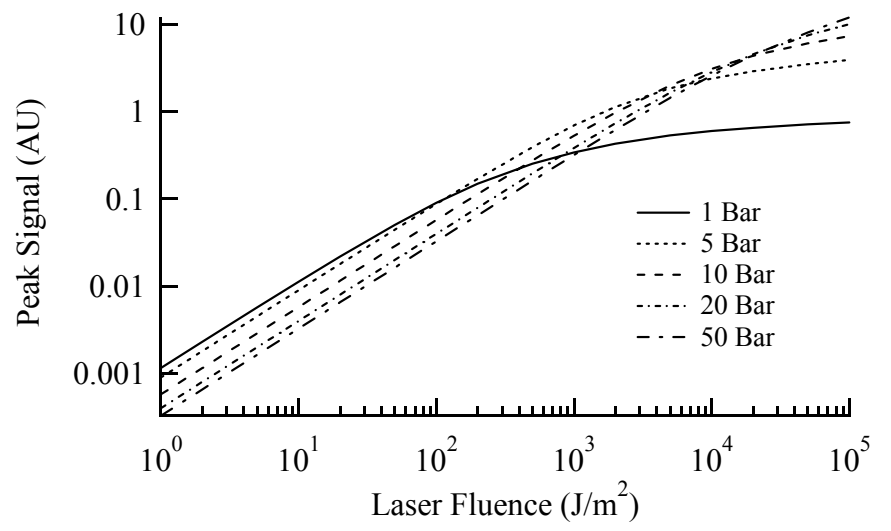

Figure 7 - Peak LIF Signal; EET all Lost

\section{Effect of Final State Distribution Following Quenching}

Two cases were run to explore the limiting effect of final state distributions following EET; all EET lost, and all EET goes to the ground vibrational state. The time dependent population of the laser excited Astate $\mathrm{v}^{\prime}=0$ level is plotted in Figure 8 for the case of 1 Bar. As can be seen, the populations (and thus fluorescence signal) are strongly influenced by the final state distribution following quenching. The time dependent populations of the X-state $\mathrm{v}$ " $=0$ level are likewise plotted in Figure 9. When all the quenched population from the A-state is lost, laser excitation depletes the population of the ground state (Figure 9). The population in the $\mathrm{v} "=1$ and 2 states decay to the ground state, but so slowly they have little effect on the outcome and the $\mathrm{v} "=0$ population is thus severely depleted over the 20 nsec gate width.

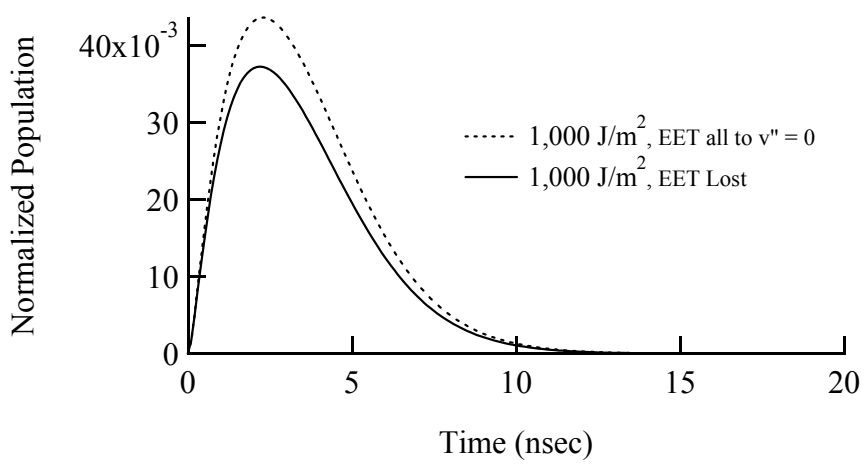

Figure 8 - 1 Bar A-state v' $=0$ Population-Time History. Effect of EET Model

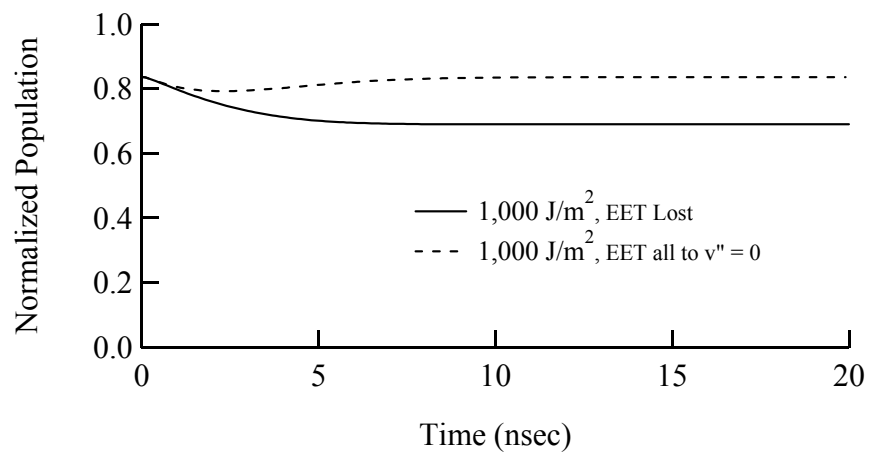

Figure $9-1$ Bar X-state v" $=0$ Population-Time History. Effect of EET Model

In contrast, when all the quenched population from the A-state is returned directly to the $\mathrm{v} "=0$ state, the population, while depleted during the high intensity portion of the pulse, rapidly recovers as A-state population decays directly back down. Upon inspection, it can be seen that only for the case where all the quenched population from the A-state is returned directly to the $\mathrm{v"}=0$ state is the concept of a "Balanced Cross Rate" likely to be satisfied. This is confirmed in Figure 10, which shows the Balanced Cross Rate sum for the two limiting cases. The effect becomes increasingly important at higher pressures. This is shown in Figure 11, which plots the percentage integrated signal difference between the two limits at increasing pressures. 


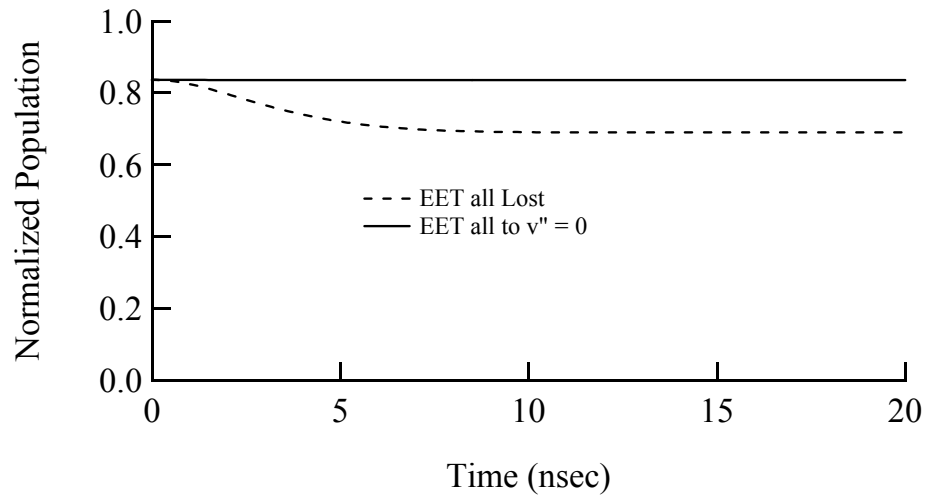

Figure 10 - 1 Bar Balanced Cross Rate Sum Time History - Effect of EET Model (Note BCS does not start out at unity because of thermal population in $\mathrm{v}$ " $=1$ and 2.)

\section{Effect of RET Rate}

To explore the effect of the RET rate on the LIF signal we carried out calculations for RET rates half and twice the base case. The results are plotted in Figure 12 for the integrated LIF signal (EET all lost). Figure 13 shows the sensitivity of the integrated LIF signal to the RET rate as a function of laser irradiance. As can be seen, there is a significant effect, peaking at $25 \%$ for a laser fluence of about $60,000 \mathrm{~J} / \mathrm{m}^{2}$. Note that the signal increases with increasing RET rate due to the faster rate at which $\mathrm{v} "=0$ population cascades into the laser coupled states.

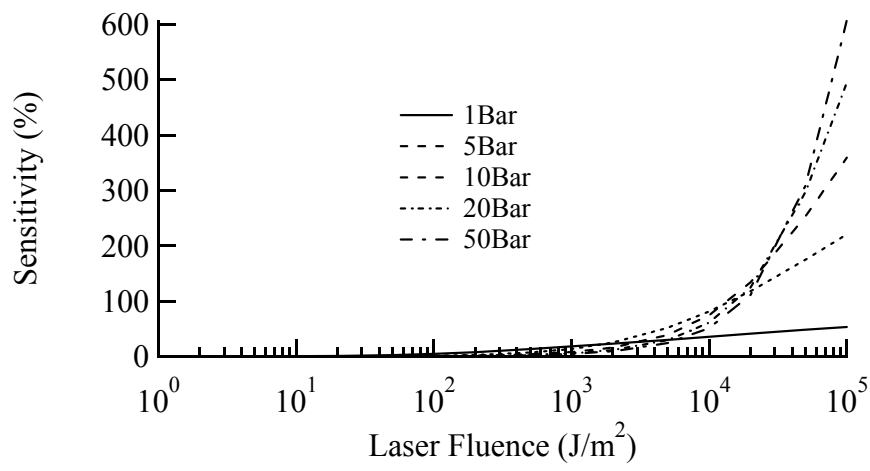

Figure 11 - Sensitivity of the Integrated LIF Signal to the final state following EET

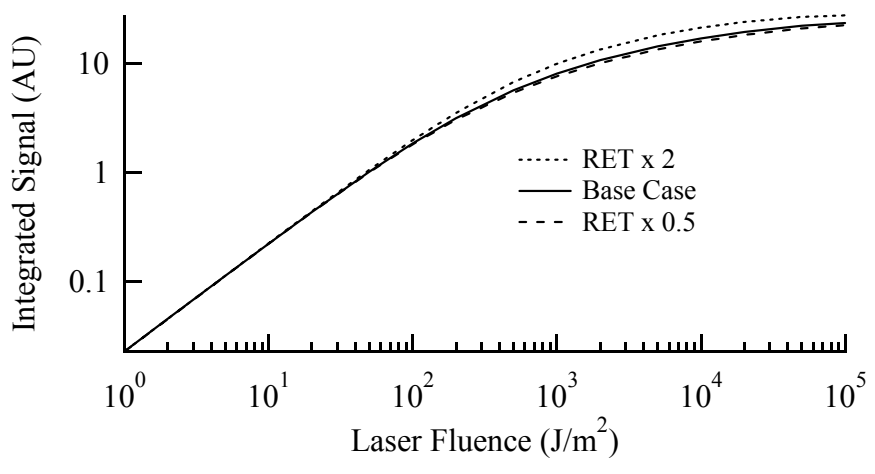

Figure 12 -Effect of RET Rate on Peak Signal

\section{E. Effect of Photoionization}

The effect of photoionization the LIF integrated signal is illustrated in Figure 14. As can be seen, ionization begins to become important at mid-level laser irradiances, and the error grows rapidly with irradiance as it is inherently a two-photon process. One photon is required to excite to the A-state, another to ionize.

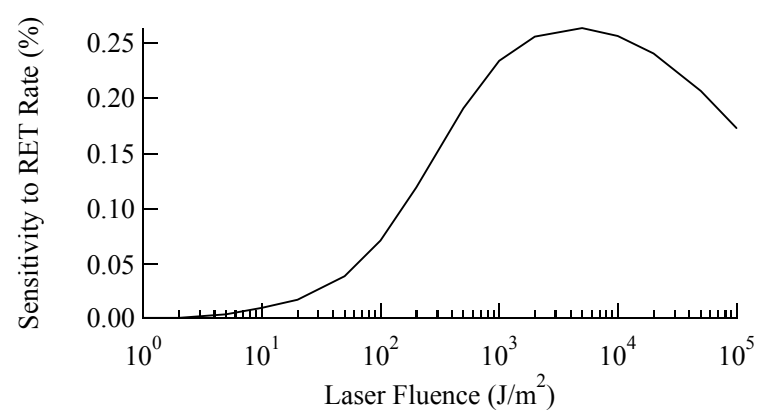

Figure 13 - Influence of RET Rate on Integrated LIF Signal - EET all Lost

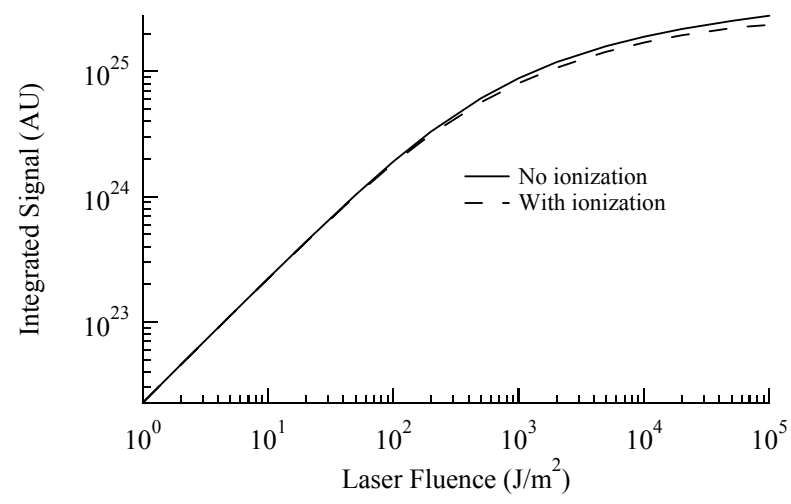

Figure 14 - Influence of ionization on LIF Signal (1 Bar) 


\section{Discussion}

When laser excitation takes place, molecules from the lower laser coupled level are excited to the upper laser coupled level. In the lower vibrational state, excitation results in depressing the population of the laser coupled rotational states. In the absence of RET, these states would rapidly become depleted, even at quite low laser irradiances. However RET rate processes try to force the population distribution back to equilibrium by cascading population into the depressed states. At very low ratios of excitation rate to RET rate, the populations remain close to their equilibrium values and are not greatly influenced by the exact value of the RET rates. In the other limit, that of very large excitation rate compared to RET rate, the populations are rapidly depleted and the outcome largely independent of the exact RET rates. However, where the two rates are comparable, then the populations, and hence overall excitation rates, become dependent on the RET rates. This is illustrated in Figure 13 showing that the effect of the RET rate peaks at a laser fluence within the range of practical interest. For the present calculation the peak roughly corresponds to the point where the two rates are approximately the same.

Depending on the excitation rate, the overall population of the ground laser-coupled vibrational state can become significantly reduced. The degree to which this occurs depends on the RET rate as discussed in the preceding paragraph, on the VET rates in the ground electronic state, and on the disposition of excited state population following quenching. As mentioned in the discussion of collisional energy transfer rates, VET rates are generally very slow. Even at combustion temperatures when a significant amount of population is initially stored in excited vibrational states, there is insufficient time for this population to return to the ground state during the laser pulse. Therefore, the only significant source for repopulation of the ground laser coupled vibrational state is quenching. This is a major effect as was shown in Figures 8-11.

The consequence of the above discussion is that depending on the specific operating conditions, the LIF signal (integrated or peak) depends not only on the quenching rate, as has been long known and accounted for, but on the RET rates and the final state disposition of quenched population. If these parameters change within an experiment (e.g. due to variations of temperature or bath gas composition) there is the potential to make a $50-100 \%$ error in estimating the total NO concentration at high laser irradiances even at one atmosphere. The effect is far greater at higher pressures due to the rapid removal of ground state population due to fast RET.

Of considerable interest is how well the various simplified models simulate the LIF signal. This will be the topic of a future paper.

\section{Summary and Conclusions}

For NO, the fastest collisional energy transfer rates are generally for quenching and RET in the Astate. Since these are approximately the same magnitude, both rotational re-distribution and quenching are important in determining the LIF spectra. For a given laser irradiance, ground state RET determines the rate at which population is cycled from the ground vibrational state to the excited state. The excited state population is determined by the ratio of the rates of ground state RET to quenching. However, the final state distribution following quenching is critically important. If quenched population is returned directly to the ground laser-coupled vibrational state, then the total population cycled through the excited state is increased, as is the LIF signal. X-state VET is very slow and there is little VET taking place during a typical laser pulse of several nanoseconds. A-state VET is fairly fast (although slower than EET and RET), so that some downward VET takes place if the $\mathrm{v}^{\prime}=1$ or higher vibrational state is excited. However, if the $\mathrm{v}^{\prime}=0$ state is excited there is little upward VET during the laser pulse.

The results show that the LIF signal is strongly dependent on the final state distribution following quenching, and to lesser, but still significant degree, on ground state RET. An important concern is that there is insufficient data for these rates. There is essentially no data on the final state distribution following quenching and little for X-state RET rates, especially at higher temperatures and for collision partners important in combustion applications. In addition, when operating at higher irradiances, any model must account for photoionization.

\section{Acknowledgements}

JWD is grateful for support from the Physikalisch-Chemisches Institut, University of Heidelberg, DAAD (Deutscher Akademischer Austauschdienst) for work carried out at Heidelberg, and the U.S. Department of Energy, Office of Basic Energy Sciences, Chemical Sciences, Geosciences, and Biosciences Division for work carried out at Sandia National Laboratories. Support for this work for VS was provided through grants from the National Science Foundation (CTS 0087337 and INT 0089227). WGB and CS acknowledge funding by DFG(Deutsche Forschungsgemeinschaft). TS acknowledges support from the U.S. De- 
partment of Energy, Office of Basic Energy Sciences, Chemical Sciences, Geosciences, and Biosciences Division.

\section{References}

1. Wolfrum, J., Proc. Comb. Inst. 27, 1 (1998).

2. Kohse-Hoinghaus, K., Progress in Energy and Combustion Science 20, 203 (1994).

3. Daily, J.W., Progress in Energy and Combustion Science 23, 133-199 (1997).

4. Bessler, W.G., C.Schulz, V. Sick and J. W. Daily, "A versatile modeling tool for nitric oxide LIF spectra," In the Proceedings of the Third Joint Meeting of the U.S. Sections of The Combustion Institute (http://www.pci.uni-heidelberg.de/pci/lifsim) (2003).

5. Pine, A.S., J.W.C. Jones and A.G. Robiette, J. Mol. Spec. 74, 52-69 (1979).

6. Amiot, C., R. Bacis and G. Guelachvili, Can. J. Phys. 56, 251-265 (1978).

7. Brown, J. M., T. J. Hougen, K. -P. Huber, J. W. C. Johns, I. Kopp, H. Lefebvre-Brion, A. J. Merer, D. A. Ramsey, J. Rostas and R. N. Zare, "The Labeling of Parity Doublet Levels in Linear Molecules," J. Mol. Spec. 55, 500 (1975).

8. Hund, F. Zeits. F. Physik 36, 657 (1926); 42, 93 (1927).

9. Van Vleck, J.H., Phys. Rev. 33, 467-506 (1929).

10. Mulliken, R.S., Rev. Mod. Phys. 2, 60 (1930).

11. Freedman, R., and R.W. Nicholls, J. Mol. Spec. 83, 223-227 (1980).

12. Engleman, R., P.E. Rouse, H.M. Peek and V.D.

Baiamonte, "Beta and Gamma Systems of Nitric Oxide, "Los Alamos Scientific Laboratory Report LA-4364, Los Alamos, NM (1970).

13. Laux, C. O. and Kruger, C. H., J. Quant. Spectrosc. Radiat. Transfer 48, 9 (1992).

14. Chang, A. Y., DiRosa, M. D., and Hanson, R. K., J. Quant. Spectrosc. Radiat. Transfer 47:375 (1992).

15. DiRosa, M. D. and Hanson, R. K., J. Mol. Spectrosc. 164:97 (1994).

16. DiRosa, M. D. and Hanson, R. K., J. Quant. Spectrosc. Radiat. Transfer 52:515 (1994).

17. Vyrodov, A. O., Heinze, J., and Meier, U. E., J. Quant. Spectrosc. Radiat. Transfer 53, 277 (1995).

18. Islam, M., I.W.M. Smith and M.H. Alexander, Phys. Chem. Chem. Phys. 2, 473 (2000).
19. Ebata, T., Y. Anezaki, M.Fujii, N. Mikami and M. Ito, Chemical Physics 84, 151 (1984).

20. Wysong, I.J., J. Chem. Phys. 101, 2800 (1994).

21. Paul, P. H., Carter, C. D., Gray, J. A., Jr., J. L. D., Thoman, J. W., and Furlanetto, M. R., Sandia Report SAND94-8237 UC-1423, Sandia National Laboratory, Livermore, CA (1994).

22. Settersten, T.B., B. Patterson, J. Gray, 3rd Joint Meeting of the US Sections of the Combustion Institute, Chicago, Ill, 2003 (The Combustion Institute).

23. Zacharias, H., R. Schmiedl and K.H. Welge, Appl. Phys. 21, 127-133 (1980).

24. Zacharias, H., H. Rottke and K.H. Welge, Appl. Phys. 24, 23-28 (1981).

25. Zacharias, H., F. de Rougemont, T.F. Heinz and M.M.T. Loy, J. Chem. Phys. 105, 111 (1996).

26. Bessler, G.W., C. Schulz, T. Lee, J. B. Jeffries, and R. K. Hanson, Appl. Opt. 41, 3547-3557 (2002). 